Article

\title{
Do Not Let the Robot Get too Close: Investigating the Shape and Size of Shared Interaction Space for Two People in a Conversation
}

\author{
Peter A. M. Ruijten * ${ }^{D}$ and Raymond H. Cuijpers \\ Department of Industrial Engineering \& Innovation Sciences, Eindhoven University of Technology, \\ 5612 AZ Eindhoven, The Netherlands; r.h.cuijpers@tue.nl \\ * Correspondence: p.a.m.ruijten@tue.nl; Tel.: +31402475213
}

Received: 23 January 2020; Accepted: 04 March 2020; Published: 6 March 2020

\begin{abstract}
Robotic and other autonomous systems that need to operate in environments with people should respect social rules. One important aspect of this is personal space, which is the space surrounding a person. When two people are in a conversation, they position themselves such that a so-called shared interaction space is created in the middle of them. The aim of the current research was to experimentally investigate the shape and size of this shared interaction space in different formations. In three experiments, we had a robot approaching two people who were having a conversation from 5 different directions, and those people indicated what would be a comfortable distance for the robot to stop. We expected that people would take the personal space of their conversation partner into account when stopping the robot. Findings of the three studies mostly confirm this expectation. Apart from some exceptions, people tend to stop the robot at a similar distance from their conversation partner as from themselves. If these findings are applied in the behavior of robotic and other autonomous systems, people would be more likely to trust and later accept robots in their physical space, ultimately creating natural social interactions between humans and robots.
\end{abstract}

Keywords: personal space; shared interaction space; comfortable distance; robot navigation

\section{Introduction}

More and more robotic and autonomous systems that need to operate in environments with people are being developed. Examples include automated cleaning systems [1], robots as receptionist [2], and robots as museum tour guides [3]. All these systems operate in a space where they not only encounter humans but also need to interact with them. In order to be able to operate in such environments, a robot's movements and interactions should be such that they respect social rules $[4,5]$.

When people, both experienced and novice users, encounter robots in public spaces, it is important that they feel comfortable in the proximity of the robot and that they accept it in their environment. As a minimum, a robot should have sophisticated social skills that allow them to adapt to social conventions [6]. One of such skills is the ability to navigate in a human- and robot-friendly way [7], which means that they should respect the distance from the person that is appropriate for the type of interaction. Different types of distances could affect people's experiences with robots. For example, Kim and Mutlu [8] studied effects of task distance (cooperation or competition), power distance (robot has the role as supervisor or assistant), and proxemic distance (physical closeness between human and robot) and found that the three types of social distances seem to influence each other. However, it is not only the distance but also its navigational behavior that seem to influence people's experiences with robots. More specifically, when a robot shows passive navigation behavior (i.e., waiting to let people 
cross their path), it is seen as more socially present than when it shows more assertive navigation behavior (i.e., passing in front of approaching people) [9]. In the current work, we focus on proxemic distance, which is strongly related to the concept of personal space.

The concept of personal space as introduced by Hall [10] divides the space surrounding a person into separate zones for different types of social interactions: the intimate zone $(<0.45 \mathrm{~m})$, the personal zone $(0.45 \mathrm{~m}-1.2 \mathrm{~m})$, the social zone $(1.2 \mathrm{~m}-3.6 \mathrm{~m})$, and the public zone $(>3.6 \mathrm{~m})$. In Hall's [10] model, each zone is represented with a circle but it may be shaped differently. For example, personal space can be egg-shaped, with distances larger in front of a person than behind [11], or it may be ellipse-shaped, with distances larger in the direction of motion [12]. Accumulating evidence suggests that robots need to stay clear from the intimate zone because entering this zone could be perceived as uncomfortable [13]. This is however not always the case, as one study showed that people tend to show compensatory behavior when a robot invades their personal space [14]. When the task requires the robot to approach a person closely, for example, when it hands over specific items or addresses a person, a robot should position itself respectively in the personal [15] and social zones [16].

\subsection{Robot Navigation and Evaluation}

Several studies have investigated the optimal interaction distance between a human and a robot. Some of these had people approach a robot to the closest comfortable distance $[17,18]$, showing that people stopped their approach at a distance between $0.45 \mathrm{~m}$ (the intimate zone) and $3.6 \mathrm{~m}$ (the social zone). Differences in stopping distance seem to result from the context of the interaction. For example, people approach a silent robot more closely than a non-silent one [18] and they approach a robot more closely when they need to hand over an object [19]. Other factors that may influence stopping distances between people and robots could be the extent to which the robot is likable [20] and the robot's appearance and behavior [21].

A paradigm that is used by many studies is to have a robot approach people from different directions. For example, Syrdal and colleagues [22] made a robot approach people until it stopped at a 20-centimeter distance and found that approaches from the front left or front right were preferred. Similar results were found by Dautenhahn and colleagues [23], who had a robot approach people until it stopped at a distance of about $50 \mathrm{~cm}$.

Most studies discussed so far used an approach distance based on Hall's [10] classification of social distances. A different approach was followed by Walters and colleagues [17]. They had a person approach a robot until they felt that the distance between them and the robot was comfortable. In a similar experiment, Torta and colleagues [16] made a robot approach a seated or standing person from 5 different directions of approach until the person pressed a button to stop the robot at a comfortable distance to start a conversation. Similar to earlier studies, people preferred approaches from the front over approaches from the side. The optimal distances that were found varied between $1.6 \mathrm{~m}$ and $1.8 \mathrm{~m}$, which is much larger than the distances used in earlier studies. An important difference was the nature of the approach. Where earlier studies mostly focused on handover tasks, this one focused on initiating a conversation.

\subsection{Interactions Involving Multiple People}

So far, we have only discussed studies that investigated preferable stopping distances and directions for interactions between one person and one robot. Ball and colleagues [24] used the same paradigm, but they also investigated whether differences occurred between people who were alone versus those who were in a dyad. People in this study had to solve a 3D jigsaw puzzle while the robot approached them from 8 possible directions, after which they would rate their perceived level of comfort. Results showed that the presence and position of a second person influenced the other person's comfort profile, indicating that people have a different comfort experience while being alone versus being together with another person. 
Similarly, Karreman and colleagues [25] investigated how two people who were placed in different formations evaluated the approach of a robot from 8 different directions while playing a Mikado game. They found differences in preference between different configurations. Like in Reference [16], people preferred an approach from the front, but only when standing in a side-by-side configuration. When standing in a face-to-face or V-shaped configuration, no clear preference of approach direction was found.

\subsection{Human Formation Patterns}

In the previous section, we introduced the concept of formation patterns, which refers to the different formations in which two or more people can stand while having a conversation. Kendon [26,27] investigated these human formation patterns and introduced the concept of o-space, which represents the focus of interaction of a group of people. When people have group conversations, they tend to place themselves in a circle facing each other. Kendon's o-space is the space in the middle of that circle. A behavior-based model for a robot that joins a group of people who are in a conversation was implemented by Althaus and colleagues [28]. While this model nicely describes the interaction spaces of groups of people, it does not work as well for dyads. For dyadic interactions, Ciolek and Kendon [26] describe different configurations that vary from closed (facing each other) to open (standing abreast). A robot that approaches two people in an interation should position itself depending on the shape and size of their shared interaction space. Although some models for describing shared interaction space have been developed [29,30], experimental validation of these models in terms of perceived comfort is largely missing.

\subsection{Research Aims}

The aim of the current research is to experimentally study the shape and size of the shared interaction space of two people in a conversation. Torta and colleagues [16] developed a paradigm to directly measure the optimal distance of approach. We used this paradigm for measuring optimal stopping distances for two people in a conversation. Being in a conversation with another person creates a shared interaction space, or o-space [27]. According to Rios-Martinez [30], this shared interaction space lies between these people, shifted slightly toward the open side. Therefore, we hypothesized that people would take the personal space of their conversation partner into account when stopping the robot. In other words, they are expected to stop the robot at the edge of the shared interaction space instead of at the border of their own personal space. In addition, we expected that stopping distances would differ between different angles of approach, with stopping distances being larger for approach angles close to zero (approaches from the front) than for ones away from zero (approaches from the side).

Data from three studies will be used. In all studies, a Nao robot (Aldebaran Robotics, Fr.) approached two people from 5 different directions until it was stopped by both people. In the first study, we explored optimal stopping distances between two people sitting next to each other in an I-shape formation [26]. In the second study, we repeated the first study, but this time, people were sitting next to each other in an L-shape formation [26]. In the third study, we repeated the second study, but this time the robot approached three different targets. Like in the earlier studies, it approached the midpoint between the two people but it also approached a point directly in front of each of the two people.

\section{Study 1}

In this study, we tested our expectation that people take the personal space of their conversation partner into account in an I-shape formation [26]. This means that people face each other while having a conversation. During the conversation, a robot will be approaching them from different angles until it is stopped at a comfortable distance by both people. 


\subsection{Method}

\subsubsection{Participants and Design}

Twenty-four participants (14 males and 10 females; $M_{\text {age }}=21.3, S D_{\text {age }}=2.03$, Range $=19$ to 26 ) were recruited for this experiment. Seven participants had previous experience with the Nao robot, but this did not significantly influence their responses. The participants performed the experiment in dyads. If there was only one participant available at a time, one of the experimenters played the role of the other participant. The experimenter in this case always pressed the button directly after the robot started walking. In addition, all stopping distance data of the experimenter were removed before analyses. The experiment lasted about $15 \mathrm{~min}$, and participants were compensated for their participation with study credits.

The robot approached participants from five different angles and headed to a point in the middle of their chairs until both participants had pressed a button to indicate that the robot reached a comfortable distance to join the conversation. After each approach, both participants evaluated that specific approach. There were 5 trials that were presented in random order.

\subsubsection{Materials}

The experiment was conducted with the Nao robot in a $6 \times 6 \mathrm{~m}$ lab at our university that was empty apart from two chairs in the center and two tables with a computer at the side. The chairs were arranged in an I-shape formation [26] and placed $1.25 \mathrm{~m}$ apart at a $90^{\circ}$ orientation. Figure 1 shows a picture and a schematic overview of the experimental setup. This figure shows the starting locations with points on a polar grid at a distance of $2 \mathrm{~m}$ from the origin and at angles of $-70^{\circ},-35^{\circ}, 0^{\circ},+35^{\circ}$, and $+70^{\circ}$. The front legs of the chairs were placed at $-0.5 \mathrm{~m}, 0 \mathrm{~m}$ and at $+0.5 \mathrm{~m}, 0 \mathrm{~m}$ from the origin of the polar grid. The resulting distance of the chair centers (representing the participant locations) from the target locations was $30 \mathrm{~cm}$.

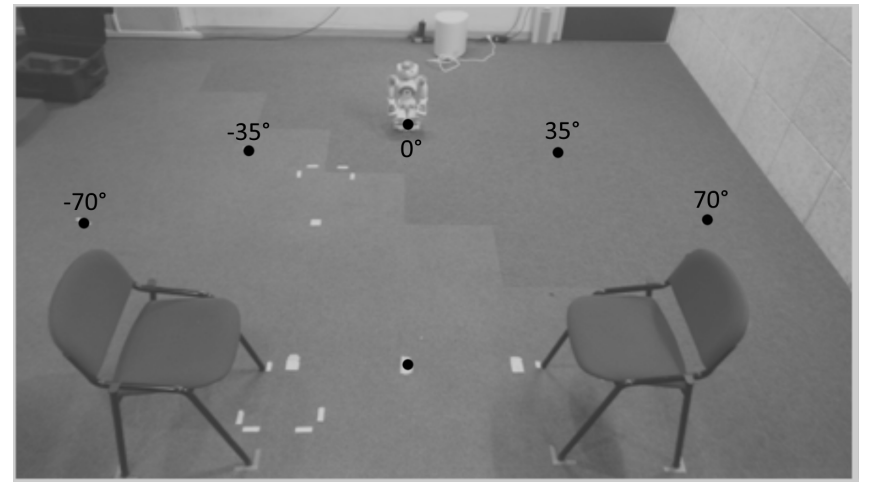

(a)

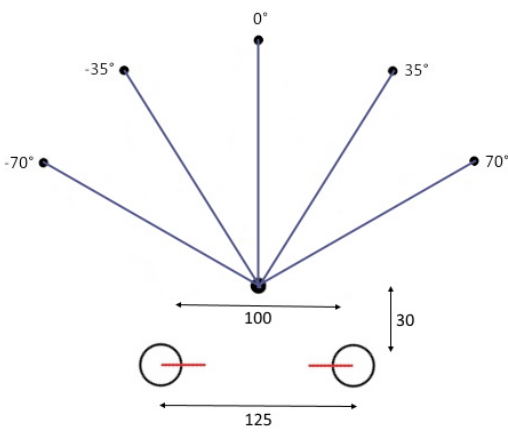

(b)

Figure 1. This figure shows (a) a picture and (b) a schematic overview of the setup in the lab for study 1. The two chairs are placed in a face-to-face (I-shape) formation. The starting points of the robot are indicated with black dots on the circular plane around the chairs. The target location of the robot is indicated with the black dot between the chairs.

The room was equipped with an Impulse X2 motion tracking system (PhaseSpace Inc., San Leandro, CA, USA) consisting of 8 cameras mounted on the walls and a red LED with wireless LED controller. This LED was attached to the robot's head for tracking its position during the experiment. The robot and the motion tracker were operated from a desktop pc running Vizard 4.0 (WorldViz, Santa Barbara, CA, USA). Two custom-built buttons for recording the stopping distances were attached to the desktop pc via a Phidget USB interface (Phidgets Inc., Calgary, AB, Canada). 


\subsubsection{Procedure}

Upon entering the lab, participants read and signed an informed consent form informing them about the aim and procedure of the study. They were informed that they would be indicating comfortable distances for interacting with the Nao robot. More specifically, the instruction was that they would be having a conversation with each other while a robot approached them. They were asked to press the button when they felt that the robot was at a comfortable distance to join the conversation. At this point, the robot, that was sitting in the corner of the lab, was shown to them.

Participants then completed two anthropomorphism scales that were part of a different study. Data on these scales will not be reported. Next, participants were seated on the chairs. In order to create a natural conversation between participants, they were told to discuss their favourite holiday destinations and to not pay special attention to the robot during its approach. While the conversation was getting on the way, a random order of trials was generated, after which one of the experimenters positioned the robot at the first starting position.

The robot approached its target location until both participants had pressed their button or until the target was reached. After both participants had pressed their button, Nao took a few more steps. This was to prevent participants from being aware when the other person pressed the button. Next, Nao returned to a crouch position and asked participants to "please rate this direction of approach". This form had one question: "How would you evaluate the approach of the robot?". It had to be answered on a 5-point scale ranging from "very bad" to "very good".

While participants answered this question, one of the experimenters positioned the robot at the next starting point. This procedure was repeated a total of 5 times. At the end of the experiment, participants again completed the two anthropomorphism scales and provided demographic information. Finally, they were thanked for their contribution and debriefed.

\subsection{Results}

Unless stated otherwise, we used linear mixed model to analyze effects of the angle of approach on stopping distance and evaluation of the approach. Prior to further analyses, we checked whether previous experience with the Nao robot influenced any of the variables of interest. Previous experience with the Nao robot influenced neither stopping distance $(F(1,94)=1.22, p=0.27)$ nor evaluation of approach $(F(1,94)=0.04, p=0.84)$. We therefore did not remove any data from further analyses.

\subsubsection{Evaluation of the Direction of Approach}

In Figure 2, average scores for participants' evaluation of approach are shown as a function of the angle of approach. A significant effect of angle of approach emerged, with $F(4,86)=3.31, p=0.014$, $\omega^{2}=0.26$. The average rating was highest for an approach angle of $0^{\circ}(M=3.85 \pm 0.19)$. The average rating was lowest for an approach angle of $-70^{\circ}(M=2.90 \pm 0.21)$. There was no significant difference between the two seats $(F(1,86)=1.08, p=0.30)$, indicating that participants who were seated on the left and right chairs evaluated the approach of the robot similarly. There was a significant interaction between the angle of approach and the seat, with $F(4,86)=4.38, p=0.003, \omega^{2}=0.26$. Figure 2 clearly shows that this effect is caused mostly by the low evaluation of the person who was seated in the right chair for a robot coming from an approach angle of $+70^{\circ}$. 


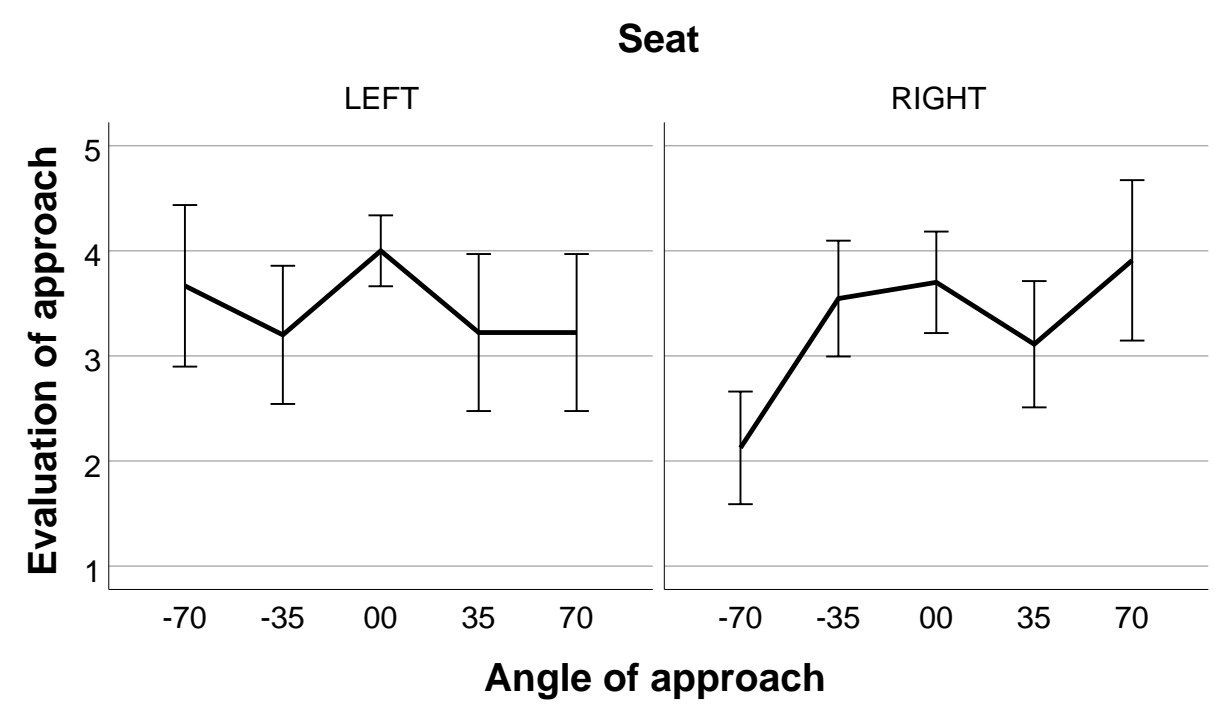

Figure 2. Average evaluation ranging from 1 (very bad) to 5 (very good) per angle of approach (ranging from $-70^{\circ}$ to $70^{\circ}$ ) and seat in study 1: Whiskers represent $95 \%$ confidence intervals.

\subsubsection{Stopping Distance}

In Figure 3, average stopping distances are shown as a function of angle of approach. A significant effect of angle of approach emerged, with $F(4,86)=4.21, p=0.004, \omega^{2}=0.22$. The average stopping distance was highest for an approach angle of $+35^{\circ}(M=1.39 \pm 0.07)$. The average stopping distance was lowest for an approach angle of $-70^{\circ}(M=1.05 \pm 0.07)$. A significant effect of seat emerged, with $F(1,86)=12.13, p=0.001, \omega^{2}=0.18$. Participants who were seated on the left chair $(M=1.36 \pm 0.04)$ on average stopped the robot at a larger distance than those who were seated on the right chair $(M=1.15 \pm 0.04)$. There was no significant interaction between angle of approach and seat $(F(4,86)=1.28, p=0.29)$.

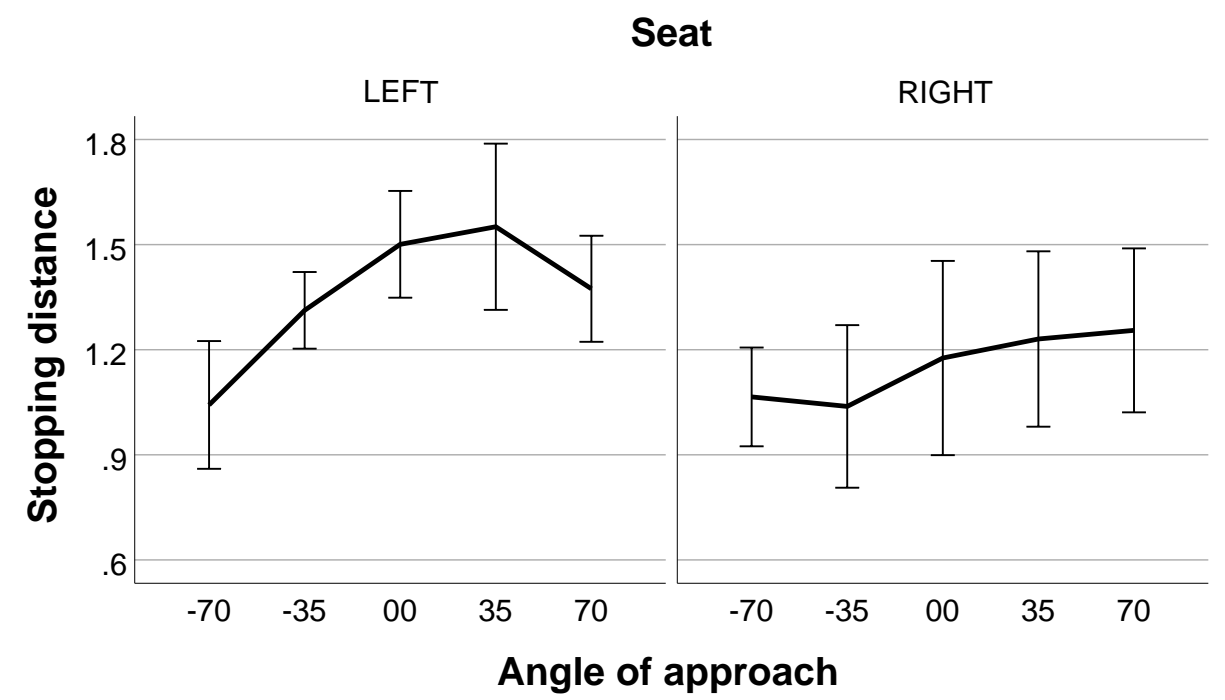

Figure 3. Average stopping distance (in meters) per angle of approach (ranging from $-70^{\circ}$ to $+70^{\circ}$ ) and seat in study 1: Whiskers represent $95 \%$ confidence intervals.

\subsection{Discussion}

An experiment was performed that explored optimal stopping distances in a shared interaction space between two people facing each other in an I-shape formation [26]. We hypothesized that people 
would stop the robot at the edge of the shared interaction space instead of at the border of their own personal space. We also expected stopping distances to differ per approach angle, with stopping distances being larger for approach angles close to zero than for ones away from zero.

Results show similar stopping distances for most approach angles, but stopping distances are bigger for large positive angles for the person sitting on the left chair. This means that those people stopped the robot at a larger distance when it approached from behind the other person. The differences in stopping distance are smaller for people sitting on the right chair. With an average difference of $20 \mathrm{~cm}$ between the two chairs, this is a rather big effect. A possible explanation for this could be the layout of the lab, in which people sitting on the right chair were closer to a wall than those sitting on the left chair (because that was where the computer controlling the robot was positioned). Having a bigger overview of the available space could have encouraged people sitting on the right chair to allow the robot to come closer.

People's evaluations of all angles of approach were rather similar except for the largest negative angle of approach for the person sitting on the right chair. This is the situation in which the robot approached them from almost straight ahead. This finding contradicts those in which a frontal approach is preferred [16], but it corresponds to earlier ones that show that people dislike robots approaching them from the front $[23,31]$. The question remains why this effect only seemed to occur for people sitting on the chair on the right. Clearly more data are needed before any conclusions can be drawn.

Our main finding that people seem to take the personal space of the other person into account was promising, but more work was needed to validate this claim. In study 2, we aimed to test whether the shape of the interaction space is different for a different formation.

\section{Study 2}

In this study, we repeated the first study, but this time, people were sitting next to each other in an L-shape formation [26]. We hypothesized that the seating arrangement of people would not influence optimal stopping distances and that people would stop the robot at the edge of the shared interaction space.

\subsection{Method}

\subsubsection{Participants and Design}

Forty-five participants (21 males and 24 females; $M_{\text {age }}=21.1, S D_{\text {age }}=1.34$, Range $=19$ to 25 ) were recruited for this experiment. Thirteen participants had previous experience with the Nao robot. The participants performed the experiment in dyads. If there was only one participant available at a time, one of the experimenters again played the role of a participant. Stopping distance data of the experimenter were removed before analysis. The experiment lasted about $15 \mathrm{~min}$ and was part of a bachelor's degree course.

Like in study 1 , the robot approached participants from five different angles and headed to a point in the middle of them until both participants had pressed a button to indicate that the robot reached a comfortable distance to join the conversation. After each approach, both participants evaluated that specific approach on the same question as in study 1 . There were 5 trials that were presented in random order.

\subsubsection{Materials}

The experiment was conducted with the Nao robot in the same room as study 1 . This time, however, the chairs were arranged in an L-shape formation [26] and placed $1.25 \mathrm{~m}$ apart at a $90^{\circ}$ orientation. Figure 4 shows a picture and a schematic overview of the experimental setup which was similar to that in study 1 . In this case, the front legs of the chairs were placed at $-0.5 \mathrm{~m}$, 
$-0.5 \mathrm{~m}$ and at $+0.5 \mathrm{~m},-0.5 \mathrm{~m}$ from the origin of the polar grid. The resulting distance of the chair centers (representing the participant locations) from the target locations was $80 \mathrm{~cm}$.

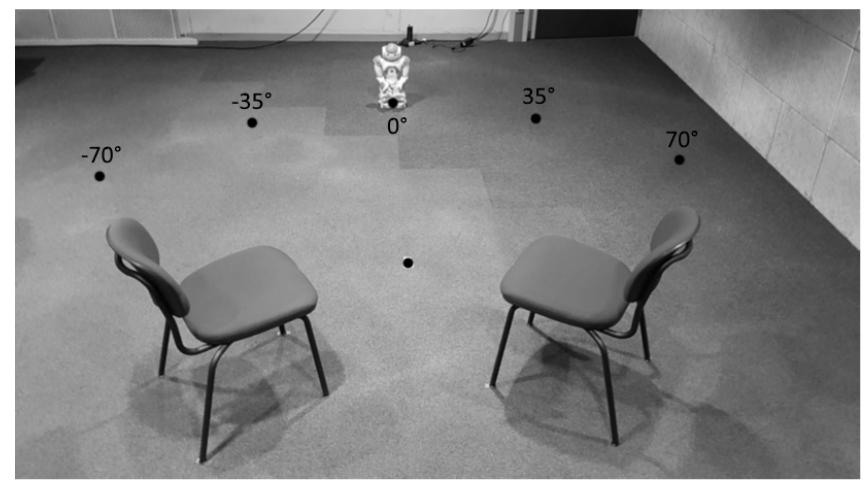

(a)

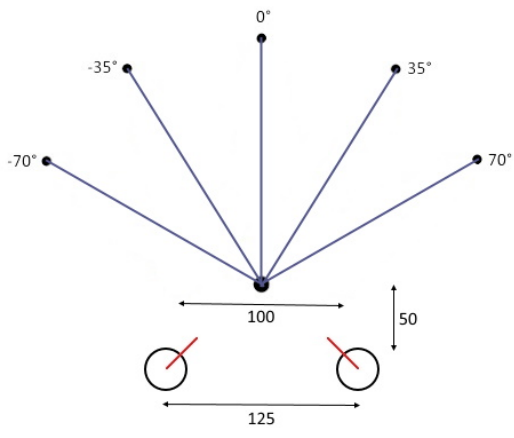

(b)

Figure 4. This figure shows (a) a picture and (b) a schematic overview of the setup in the lab for study 2. The two chairs are placed in an L-shape formation. The starting points of the robot are indicated with black dots on the circular plane around the chairs. The target location of the robot is indicated with the black dots between the chairs.

\subsubsection{Procedure}

The procedure was identical to that of study 1. Participants first read and signed an informed consent form informing them about the aim and procedure of the study. Participants then completed the two anthropomorphism scales (which were again used for different purposes) and were seated on the chairs. They received the same instructions about the robot approaching them and were again asked to stop it at a distance that was comfortable for joining the interaction. While conversing about holiday destinations, the robot approached them from each of the 5 different angles in random order. Participants evaluated each of the approaches with the same question as in study 1 . At the end of the experiment, participants again completed the two anthropomorphism scales and provided demographic information. They were finally thanked for their contribution and debriefed.

\subsection{Results}

Unless stated otherwise, we again used linear mixed models to analyze effects of approach direction on stopping distance and evaluation of the approach. Again, previous experience with the Nao robot influenced neither stopping distance $(F(1,188)=0.26, p=0.61)$ nor evaluation of approach $(F(1,188)=0.13, p=0.72)$. We therefore did not remove any data from further analyses.

\subsubsection{Evaluation of the Direction of Approach}

In Figure 5, average scores for participants' evaluation of approach are shown as a function of angle of approach. A significant effect of angle of approach emerged, with $F(4,180)=3.63$, $p=0.007, \omega^{2}=0.16$. The average rating was highest for an approach angle of $+35^{\circ}(M=3.46 \pm 0.14)$. The average rating was lowest for an approach angle of $-70^{\circ}(M=2.82 \pm 0.15)$. There was no significant difference between the two seats $(F(1,180)=0.92, p=0.34)$, indicating that participants who were seated on the left and right chairs evaluated the approach of the robot similarly.

There was a significant interaction between angle of approach and seat, with $F(4,180)=5.14$, $p=0.001, \omega^{2}=0.16$. This interaction is clearly visible in the figure, which shows that evaluations of participants who were seated on the left chair were more negative for large positive angles, while those of participants who were seated on the right chair were more negative for large negative angles. In the L-formation used in this experiment, these situations correspond to approaches from the front: $+70^{\circ}$ for participants seated on the left and $-70^{\circ}$ for participants seated on the right. 


\section{Seat}

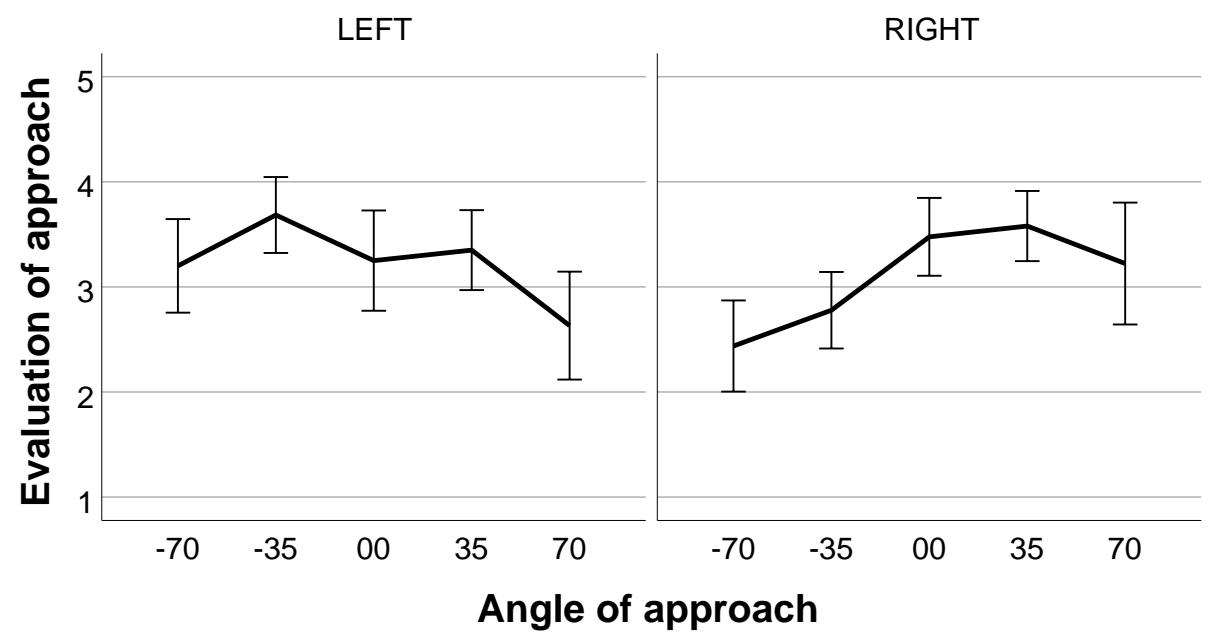

Figure 5. Average evaluation ranging from 1 (very bad) to 5 (very good) per angle of approach (ranging from $-70^{\circ}$ to $+70^{\circ}$ ) and seat in study 2: Whiskers represent $95 \%$ confidence intervals.

\subsubsection{Stopping Distance}

In Figure 6, average stopping distances are shown as a function of angle of approach. A significant effect of angle of approach emerged, with $F(4,180)=11.70, p<0.001, \omega^{2}=0.22$. The average stopping distance was highest for an approach angle of $+35^{\circ}(M=1.46 \pm 0.04)$. The average stopping distance was lowest for an approach angle of $-70^{\circ}(M=1.07 \pm 0.05)$. A significant effect of seat emerged, with $F(1,180)=31.05, p<0.001, \omega^{2}=0.18$. Participants who were seated on the left chair $(M=1.43 \pm 0.03)$ on average stopped the robot at a larger distance than those seated on the right chair $(M=1.21 \pm 0.03)$. There was no significant interaction between angle of approach and seat $(F(4,180)=1.62, p=0.17)$.

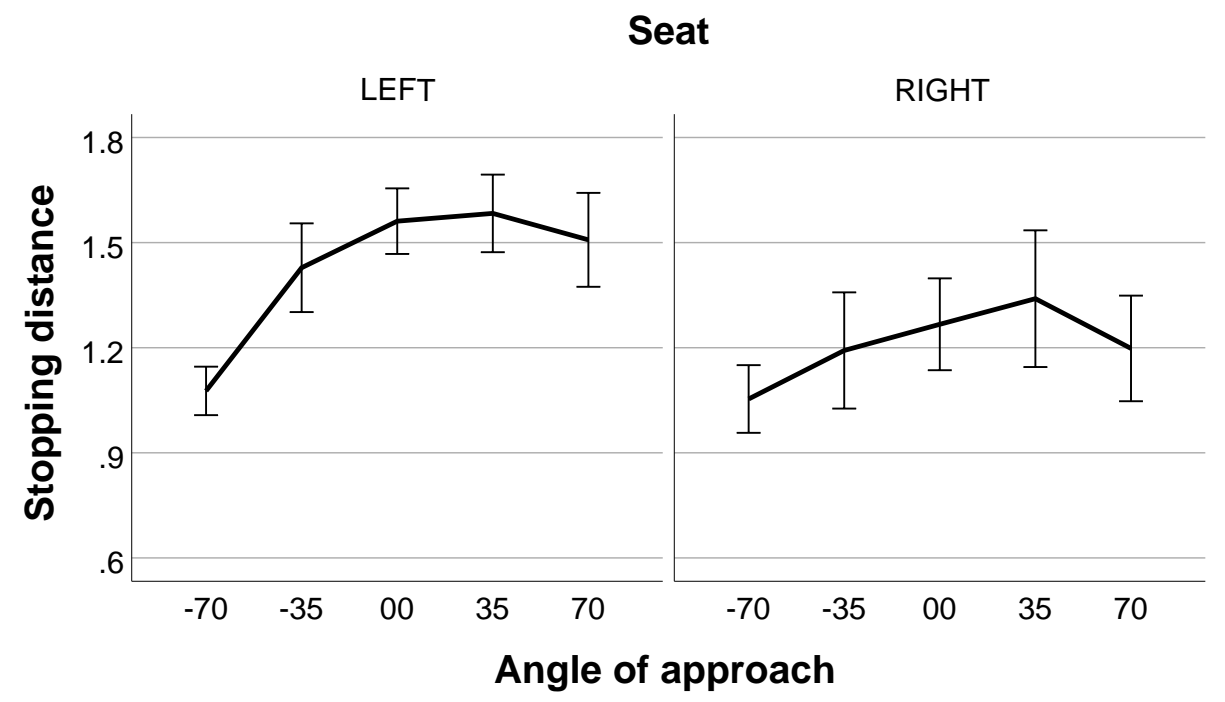

Figure 6. Average stopping distance (in meters) per angle of approach (ranging from $-70^{\circ}$ to $+70^{\circ}$ ) and seat in study 2: Whiskers represent $95 \%$ confidence intervals.

\subsection{Discussion}

This experiment explored optimal stopping distances in a shared interaction space between two people sitting next to each other in an L-shape formation [26]. We again hypothesized people to stop 
the robot at the edge of the shared interaction space instead of at the border of their own personal space. Results show that stopping distances are bigger for large positive angles than for large negative ones, especially for people sitting in the chair on the left. The average difference between the two chairs was again about $20 \mathrm{~cm}$, so the effect found in study 1 seems to be consistent.

People's evaluations of the approaching robot were more positive when it approached them from straight ahead, which is in line with findings of study 1 . This time, however, the pattern seemed to occur for all participants. Our finding in study 1 that people take the personal space of the other person into account was also validated in the current experiment. If people indeed take another person's personal space into account when stopping a robot at a comfortable distance, this would mean that different stopping distances can be expected when the robot approaches targets different than the midpoint between two people. This expectation will be tested in study 3 .

\section{Study 3}

In this study, we replicated the second study, but this time, we varied the target location of the robot to be in front of either person or directly in between. We hypothesized that the preferred stopping distance would be smaller for the person who the robot approached than for the other person in the interaction.

\subsection{Method}

\subsubsection{Participants and Design}

Thirty-two participants ( 24 males and 6 females; $M_{\text {age }}=23.5, S D_{\text {age }}=2.19$, Range $=18$ to 28 ) were recruited for this experiment. For one dyad, no data are available due to overheating problems with the robot. Of the remaining 30 participants, 21 had previous experience with the Nao robot. The participants performed the experiment in dyads. The experiment lasted about $30 \mathrm{~min}$, for which participants were paid $5 €$.

During the experiment, the Nao robot approached the participants from each of five different angles, but this time, it also headed to 3 different target locations. A picture and a schematic overview of the experimental setup with the approach angles and target points are provided in Figure 7 . This figure shows the starting locations with points on a polar grid at a distance of $2 \mathrm{~m}$ from the origin and at angles of $-70^{\circ},-35^{\circ}, 0^{\circ},+35^{\circ}$, and $+70^{\circ}$. The target locations were the origin, a point $0.5 \mathrm{~m}$ to the left of the origin, and a point $0.5 \mathrm{~m}$ to the right of the origin. Participants experienced 15 trials that were presented in random order.

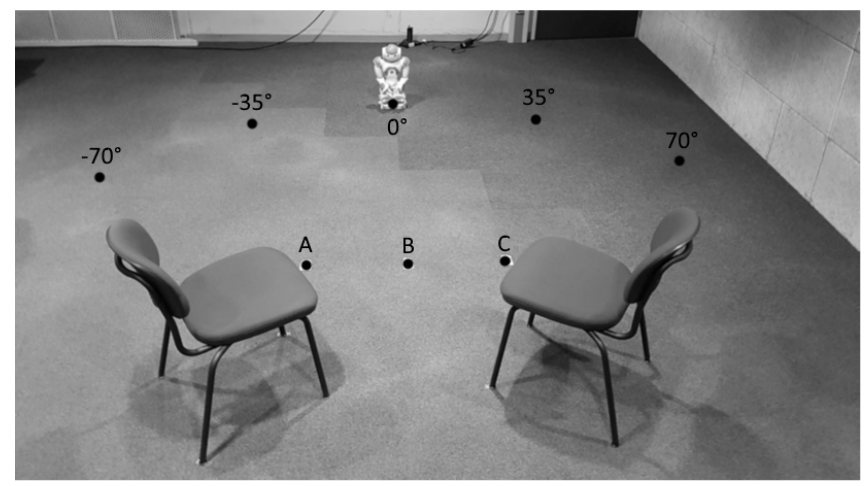

(a)

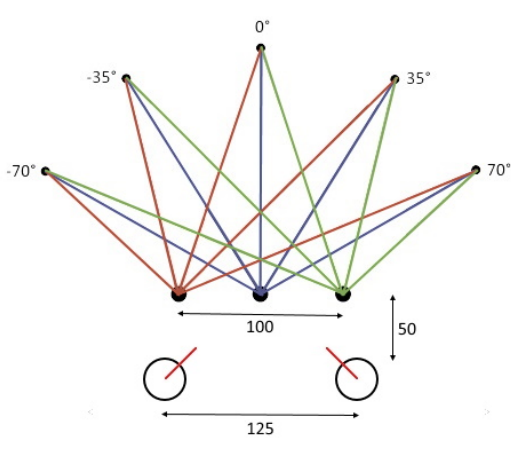

(b)

Figure 7. This figure shows (a) a picture and (b) a schematic overview of the setup in the lab for study 3. The two chairs are placed in an L-shape formation. The starting points of the robot are indicated with black dots on the circular plane around the chairs. The target locations of the robot are indicated with black dots between the chairs. 


\subsubsection{Materials}

The experiment was conducted with the Nao robot (Aldebaran Robotics, Fr.) in the same room as the other two studies. The chairs were arranged in an L-shape formation [26] and placed $1.25 \mathrm{~m}$ apart at a $90^{\circ}$ orientation, such that the front legs of the chairs were placed at $-0.5 \mathrm{~m},-0.5 \mathrm{~m}$ and at $+0.5 \mathrm{~m}$, $-0.5 \mathrm{~m}$ from the origin of the polar grid. The resulting distance of the chair centers (representing the participant locations) from the target locations was $80 \mathrm{~cm}$.

\subsubsection{Procedure}

The procedure was identical to that of the other studies. Participants signed an informed consent form, completed the anthropomorphism scales (again for different purposes than the current research), were seated in the chairs, indicated preferred stopping distance and evaluation of approach for a total of 15 times, completed the anthropomorphism scales again and provided demographic information, were thanked for their contribution, were debriefed, and were paid for their participation.

\subsection{Results}

Unless stated otherwise, we again used linear mixed model to analyze effects of approach direction on stopping distance and evaluation of the approach. Again, previous experience with the Nao robot did influenced neither stopping distance $(F(1,407)=1.45, p=0.23)$ nor evaluation of approach $(F(1,401)=1.64, p=0.20)$. We therefore did not remove any data from further analyses.

\subsubsection{Evaluation of the Direction of Approach}

In Figure 8, the average scores for participants' evaluation of approach are shown as a function of angle of approach and target. A significant effect of angle of approach emerged, with $F(4,373)=2.97$, $p=0.020, \omega^{2}=0.09$. The average rating was highest for an approach angle of $+35^{\circ}(M=3.31 \pm 0.12)$. The average rating was lowest for an approach angle of $-70^{\circ}(M=2.79 \pm 0.12)$. There was no significant difference between the two seats $(F(1,373)=1.06, p=0.30)$, indicating that participants who were seated on the left and right chairs evaluated the approach of the robot similarly. There was also no significant effect of target $(F(2,373)=1.40, p=0.25)$, showing that participants stopped the robot on average at similar distances between the three targets.

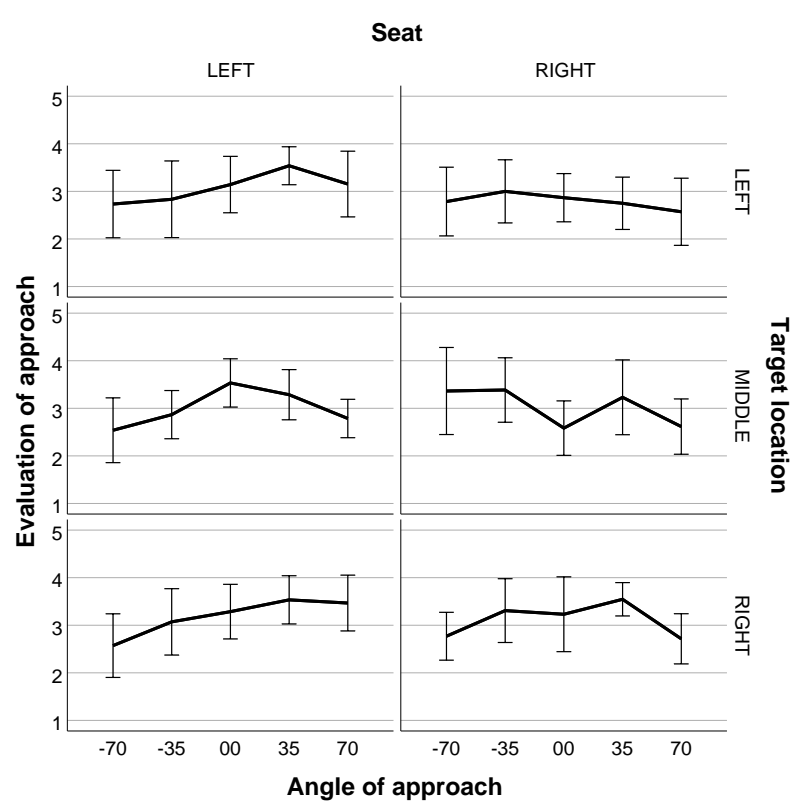

Figure 8. Average evaluation ranging from 1 (very bad) to 5 (very good) per angle of approach (ranging from $-70^{\circ}$ to $70^{\circ}$ ), seat, and target location in study 3: Whiskers represent $95 \%$ confidence intervals. 
There was a significant interaction between angle of approach and seat, with $F(4,373)=3.08$, $p=0.016, \omega^{2}=0.09$. It seems that participants seated on the left chair had a slight preference for the robot approaching from large positive angles, while no such difference is visible for participants seated on the right chair. The differences in evaluation are however smaller than in the first and second study.

Finally, there were no significant interaction effects between target and angle of approach $(F(8,373)=0.49, p=0.87)$ or between target and seat $(F(2,373)=0.80, p=0.45)$.

\subsubsection{Stopping Distance}

In Figure 9, average stopping distances are shown as a function of angle of approach and target. There was no significant effect of angle of approach $(F(1,379)=1.54, p=0.19)$. A significant effect of seat did emerge, with $F(1,379)=10.79, p=0.001, \omega^{2}=0.21$. Participants who were seated on the left chair $(M=1.10 \pm 0.02)$ on average stopped the robot at a larger distance than those who were seated on the right chair $(M=1.00 \pm 0.02)$.

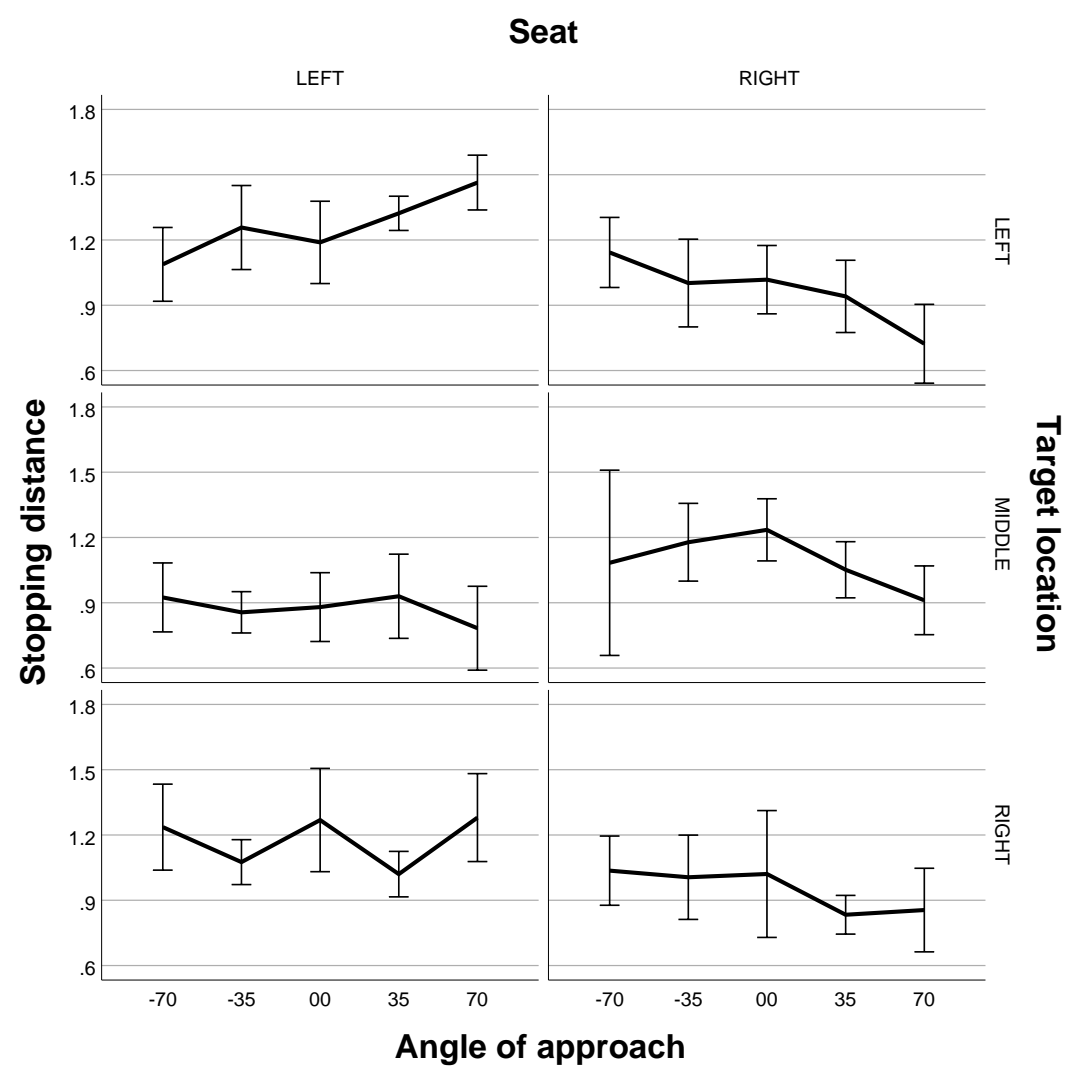

Figure 9. Average stopping distance (in meters) per angle of approach (ranging from $-70^{\circ}$ to $+70^{\circ}$ ), seat, and target location in study 3 : Whiskers represent $95 \%$ confidence intervals.

There was also a significant effect of target, with $F(2,379)=5.96, p=0.003, \omega^{2}=0.18$. Participants let the robot come closest when it approached the middle target $(M=0.98 \pm 0.03)$, followed by the target in front of the right chair $(M=1.06 \pm 0.03)$; the largest stopping distances were found when the robot approached the target in front of the left chair $(M=1.12 \pm 0.03)$.

Finally, there were significant interaction effects between angle of approach and seat $\left(F(4,379)=4.67, p=0.001, \omega^{2}=0.23\right)$ and between seat and target $(F(2,379)=23.50, p<0.001$, $\omega^{2}=0.23$ ). As can be seen in Figure 9, for each of the three target locations, participants who were seated on the left chair seemed to respond differently to various approach angles than participants seated on the right chair. When the robot approached the target close to the left chair, participants on that chair appear to allow the robot to come closer when it comes from the side of the other 
participant than when it comes from their own side. The same pattern occurred for participants who were seated on the right chair. When the robot approached the target in the middle of the two chairs, participants seated on the left chair stopped the robot at about the same distance for all approach angles, while participants seated on the right chair preferred a slightly larger distance when the robot approached from the middle. When the robot approached the target close to the right chair, participants on that chair stopped the robot at about the same distance for all approach angles while participants on the left chair stopped the robot at slightly varying distances.

\subsection{Discussion}

This experiment explored optimal stopping distances in a shared interaction space between two people sitting next to each other in an L-shape formation [26]. This time, the target location of the robot was varied to be in front of either person or directly in between. We hypothesized that the preferred stopping distance would be smaller for the person that the robot approached than for the other person in the interaction.

Results show different patterns over the three target locations. When approaching the target in front of the person on the left chair, stopping distances follow the expected pattern. That is, both people stop the robot at a larger distance when it approaches from the side of the other person than when it approaches from their own side. When the robot approaches the target in the middle of the two chairs, stopping distances are rather similar between the different approach angles, representing an equal distance from both people. These two patterns indicate that people take the personal space of the other person into account when stopping the robot.

Stopping distances did not follow this expected pattern when the robot approaches the target in front of the person seated on the right chair. In this case, people on the right chair clearly stopped the robot at a smaller distance than those on the left chair. This finding is similar to those in studies 1 and 2 and can be related to the setup of the lab. Future studies could aim to investigate this effect in more detail.

Unlike in studies 1 and 2, no obvious pattern occurred for people's evaluations of the approaching robot. A possible explanation for this could be that the duration of the current experiment was longer than that of the first two studies, making the conversations between people longer as well. As the duration of the conversation increased, their attention to the robot may have decreased a bit, leading to rather neutral evaluations for all approach angles.

\section{General Discussion}

A series of experiments was performed with the aim to experimentally validate the shape and size of the shared interaction space of two people in a conversation. We were interested in whether people would take the personal space of another person into account while being approached by a Nao robot. Our findings show that, in most cases, they do so, as seen by larger stopping distances when the robot approaches from behind the other person.

In an experiment similar to the current one [16], stopping distances between $1.6 \mathrm{~m}$ and $1.8 \mathrm{~m}$ were observed. In the current studies, we observed stopping distances ranging between about $1.0 \mathrm{~m}$ and $1.4 \mathrm{~m}$ relative to a target position that was about $0.5 \mathrm{~m}$ in front of the participants. This corresponds to a stopping distance of $1.5 \mathrm{~m}$ to $1.9 \mathrm{~m}$ and, thus, closely matches earlier work. When looking at this more closely, we could state that a group of people places themselves in circular formation oriented towards the center (see Reference [27]), which in our case would mean that people sat on a circle with radius $\mathrm{r}=88 \mathrm{~cm}\left(\frac{1}{2} \sqrt{2}\right.$ times the interpersonal distance of $\left.1.25 \mathrm{~m}\right)$. Based on the work by Kendon [27], we expected that the best position for the robot to stop is on the median line between the participants, which is at a distance of $0.88+1.25 / 2=1.50 \mathrm{~m}$. This value also closely matches our findings.

Several models for human proxemics were developed. For example, Sisbot and colleagues [32,33] developed a human-aware model for navigation that takes social acceptability into account. This model focuses on a person's field of view and posture, but it takes people as nonmoving entities. Satake 
and colleagues [34] extended on this with a three-stage model in which the robot predicts a person's walking behavior, finds a suitable person with whom to engage in an interaction, announces itself to attract that person's attention, and then initiates a conversation.

Papadakis and colleagues $[35,36]$ proposed a probabilistic model for robots to adapt their path-planning trajectories to social sensitivity levels. Finally, Charalampous and colleagues [37] developed a method for planning socially acceptable paths based on the actions a person is performing.

A nice feature of our paradigm is that we are able to measure the stopping distance of both participants of a dyad separately, allowing us to show that people in a conversation seem to stop the robot at the edge of their shared interaction space. This finding confirms that robots should take the shared interaction space into account when approaching humans that are interacting with each other.

In addition to the stopping distances, we also measured how our participants evaluated each approach. In studies 1 and 2, the most positively evaluated approaches were those from the sides where the people were sitting. This effect was rather small though, and no such clear patterns were found in the third study. A similar study that investigated perceived comfort of people in various formations found no significant effects of approach angle [25]. Another study that had a robot approach people from different directions showed a small preference for approaches from the right side [23]. More recent work that evaluated perceived comfort of a robot passing people on either their left or right sides also found no differences between these two sides [13]. Based on these findings, we cannot yet conclude that people prefer approaches from one side or another.

\section{Limitations and Future Work}

The studies in this paper were performed with a Nao robot, which is just one of the many robots that have been used for this kind of work in recent years. Walters and colleagues [38] showed that stopping distances depend on a robot's appearance and behavior and on the type of interaction people have with the robot. Different stopping distances were observed for PR2 [39], Bandit II [21], and the Nao robot [16] in earlier studies. These robots vary greatly in height and size, making it hard to generalize the findings of the current work. The notion that people seem to take the personal space of others into account should however not differ between different robots, and future work can be designed to validate whether this is indeed the case.

Human positioning in relation to a robot also differs between different robot activities (handing over an object or uttering a verbal message), user activities, or the perceived capabilities of the robot [21]. With all these contextual factors playing a role, it is important to keep as many factors as possible constant. We have attempted to do so by executing three similar studies that seem to show a consistent pattern.

In our studies, participants who mostly did not know each other beforehand were instructed to start a conversation about their favourite holiday destinations, which could have been awkward for them given the interruptions from questionnaires and the repositioning of the robot. Our experiences with these participants was nevertheless rather positive, such that they seemed to have little trouble chatting about these holiday destinations. Future work on estimating where robots should place themselves to join a group interaction could make use of this topic to create natural interactions between strangers.

It was also not hard for them to figure out that we were mostly interested in the stopping distances and how they varied between the approach angles. This could have influenced their behavior in the experiments. We still believe it is unlikely that the patterns of responses were affected, since participants were probably unaware of their own personal space and their shared interaction space. Future work could test this more explicitly by asking people whether they deliberately leave more space between them and the robot when it gets close to other people in the interaction.

Finally, participants were not restricted in how they turned their heads during the experiments, and many of them were facing each other (even in the L-shaped formation). As a result, they may have looked at the robot once in a while, but looked at the other participant the rest of the time. 
While the robot is in their peripheral vision, they would still be able to see the robot while deciding to stop it. When the robot was approaching them from the back, they will probably have made their decision based on auditory signals from the robot. If people did indeed turn their heads towards each other, they essentially cancelled out the manipulation of seating configuration, which would make the differences smaller. Results however still showed differences in stopping distance between the two people, so the influence of people turning their heads is rather small.

\section{Conclusions}

The current work investigated whether people would stop a robot at the edge of their own personal space or at the edge of a shared interaction space. Findings point in the direction of the latter. This means that, if robotic and autonomous systems need to operate in environments with people, they should take this into account. We believe that robots should respect shared interaction spaces and should choose a proper orientation when engaging with multiple users. When they do, people may be more likely to trust and, later, to accept robots in their physical space, ultimately creating natural social interactions between humans and robots.

Author Contributions: Conceptualization, P.A.M.R. and R.H.C.; methodology, P.A.M.R. and R.H.C.; software, R.H.C.; validation, P.A.M.R. and R.H.C.; formal analysis, P.A.M.R. and R.H.C.; investigation, P.A.M.R. and R.H.C.; resources, P.A.M.R. and R.H.C.; data curation, P.A.M.R. and R.H.C.; writing-original draft preparation, P.A.M.R.; writing-review and editing, P.A.M.R. and R.H.C.; visualization, P.A.M.R.; supervision, P.A.M.R. and R.H.C.; project administration, P.A.M.R. and R.H.C. All authors have read and agreed to the published version of the manuscript.

Funding: This research received no external funding.

Conflicts of Interest: The authors declare no conflict of interest.

\section{References}

1. Elkmann, N.; Hortig, J.; Fritzsche, M. Cleaning automation. In Springer Handbook of Automation; Springer: Berlin/Heidelberg, Germany, 2009; pp. 1253-1264.

2. Hashimoto, T.; Hiramatsu, S.; Tsuji, T.; Kobayashi, H. Realization and evaluation of realistic nod with receptionist robot SAYA. In Proceedings of the RO-MAN 2007-The 16th IEEE International Symposium on Robot and Human Interactive Communication, Jeju, Korea, 26-29 August 2007; pp. 326-331.

3. Kanda, A.; Arai, M.; Suzuki, R.; Kobayashi, Y.; Kuno, Y. Recognizing groups of visitors for a robot museum guide tour. In Proceedings of the 2014 7th International Conference on Human System Interactions (HSI), Costa da Caparica, Portugal, 16-18 June 2014; pp. 123-128.

4. Kirby, R.; Forlizzi, J.; Simmons, R. Affective social robots. Robot. Auton. Syst. 2010, 58, 322-332. [CrossRef]

5. De Graaf, M.M.; Allouch, S.B. Exploring influencing variables for the acceptance of social robots. Robot. Auton. Syst. 2013, 61, 1476-1486. [CrossRef]

6. Fong, T.; Nourbakhsh, I.; Dautenhahn, K. A survey of socially interactive robots. Robot. Auton. Syst. 2003, 42, 143-166. [CrossRef]

7. Lam, C.P.; Chou, C.T.; Chiang, K.H.; Fu, L.C. Human-centered robot navigation-towards a harmoniously human-robot coexisting environment. IEEE Trans. Robot. 2010, 27, 99-112. [CrossRef]

8. Kim, Y.; Mutlu, B. How social distance shapes human-robot interaction. Int. J. Hum.-Comput. Stud. 2014, 72, 783-795. [CrossRef]

9. Fiore, S.M.; Wiltshire, T.J.; Lobato, E.J.; Jentsch, F.G.; Huang, W.H.; Axelrod, B. Toward understanding social cues and signals in human-robot interaction: Effects of robot gaze and proxemic behavior. Front. Psychol. 2013, 4, 859. [CrossRef] [PubMed]

10. Hall, E.T. A system for the notation of proxemic behavior. Am. Anthropol. 1963, 65, 1003-1026. [CrossRef]

11. Hayduk, L.A. The shape of personal space: An experimental investigation. Can. J. Behav. Sci. Can. Des Sci. Du Comport. 1981, 13, 87. [CrossRef]

12. Helbing, D.; Molnar, P. Social force model for pedestrian dynamics. Phys. Rev. E 1995, 51, 4282. [CrossRef] [PubMed] 
13. Neggers, M.M.; Cuijpers, R.H.; Ruijten, P.A. Comfortable passing distances for robots. In Proceedings of the International Conference on Social Roboticsm, Qingdao, China, 28-30 November 2018; Springer: Berlin/Heidelberg, Germany, 2018; pp. 431-440.

14. Sardar, A.; Joosse, M.; Weiss, A.; Evers, V. Don't stand so close to me: Users' attitudinal and behavioral responses to personal space invasion by robots. In Proceedings of the Seventh Annual ACM/IEEE International Conference on Human-Robot Interaction, Boston, MA, USA, 5-8 March 2012; pp. 229-230.

15. Walters, M.L.; Dautenhahn, K.; Koay, K.L.; Kaouri, C.; Boekhorst, R.; Nehaniv, C.; Werry, I.; Lee, D. Close encounters: Spatial distances between people and a robot of mechanistic appearance. In Proceedings of the 5th IEEE-RAS International Conference on Humanoid Robots, 2005, Tsukuba, Japan, 5 December 2005; pp. 450-455. [CrossRef]

16. Torta, E.; Cuijpers, R.H.; Juola, J.F. Design of a parametric model of personal space for robotic social navigation. Int. J. Soc. Robot. 2013, 5, 357-365. [CrossRef]

17. Walters, M.L.; Dautenhahn, K.; te Boekhorst, R.; Koay, K.L.; Kaouri, C.; Woods, S.; Nehaniv, C.; Lee, D.; Werry, I. The influence of subjects' personality traits on personal spatial zones in a human-robot interaction experiment. In Proceedings of the ROMAN 2005. IEEE International Workshop on Robot and Human Interactive Communication, Nashville, TN, USA, 13-15 August 2005; IEEE: Piscataway, NJ, USA, 2005; pp. 347-352.

18. Walters, M.L.; Syrdal, D.S.; Dautenhahn, K.; te Boekhorst, R.; Koay, K.L. Avoiding the uncanny valley: Robot appearance, personality and consistency of behavior in an attention-seeking home scenario for a robot companion. Auton. Robot. 2008, 24, 159-178. [CrossRef]

19. Koay, K.L.; Sisbot, E.A.; Syrdal, D.S.; Walters, M.L.; Dautenhahn, K.; Alami, R. Exploratory Study of a Robot Approaching a Person in the Context of Handing Over an Object. In Proceedings of the AAAI Spring Symposium: Multidisciplinary Collaboration for Socially Assistive Robotics, Palo Alto, CA, USA, 26-28 March 2007; pp. 18-24.

20. Mumm, J.; Mutlu, B. Human-robot Proxemics: Physical and Psychological Distancing in Human-robot Interaction. In Proceedings of the HRI '11: 6th International Conference on Human-Robot Interaction, Lausanne, Switzerland, 8-11 March 2011; ACM: New York, NY, USA, 2011; pp. 331-338. [CrossRef]

21. Mead, R.; Mataric, M.J. Robots Have Needs Too: People Adapt Their Proxemic Preferences to Improve Autonomous Robot Recognition of Human Social Signals. In New Frontiers in Human-Robot Interaction; John Benjamins Publishing Company: Amsterdam, The Netherlands, 2015; p. 100.

22. Syrdal, D.S.; Dautenhahn, K.; Woods, S.; Walters, M.L.; Koay, K.L. 'Doing the right thing wrong'-Personality and tolerance to uncomfortable robot approaches. In Proceedings of the ROMAN 2006-The 15th IEEE International Symposium on Robot and Human Interactive Communication, Hatfield, UK, 6-8 September 2006; pp. 183-188. [CrossRef]

23. Dautenhahn, K.; Walters, M.; Woods, S.; Koay, K.L.; Nehaniv, C.L.; Sisbot, A.; Alami, R.; Siméon, T. How may I serve you?: A robot companion approaching a seated person in a helping context. In Proceedings of the 1st ACM SIGCHI/SIGART Conference on Human-Robot Interaction, Salt Lake City, UT, USA, 2-3 March 2006; ACM: New York, NY, USA, 2006; pp. 172-179.

24. Ball, A.; Rye, D.; Silvera-Tawil, D.; Velonaki, M. Group Vs. Individual Comfort When a Robot Approaches. In Proceedings of the Social Robotics: 7th International Conference, ICSR 2015, Paris, France, 26-30 October 2015; Tapus, A., André, E., Martin, J.C., Ferland, F., Ammi, M., Eds.; Springer: Cham, Switzerland, 2015; pp. 41-50.

25. Karreman, D.; Utama, L.; Joosse, M.; Lohse, M.; van Dijk, B.; Evers, V. Robot Etiquette: How to Approach a Pair of People? In Proceedings of the HRI'14: ACM/IEEE International Conference on Human-Robot Interaction, Bielefeld, Germany, 3-6 March 2014; ACM: New York, NY, USA, 2014; pp. 196-197.

26. Ciolek, M.; Kendon, A. Environment and the spatial arrangement of conversational encounters. Sociol Inq 1980, 50, 237-271. [CrossRef]

27. Kendon, A. Conducting Interaction: Patterns of Behavior in Cocused Encounters; CUP Archive: Cambridge, UK, 1990; Volume 7.

28. Althaus, P.; Ishiguro, H.; Kanda, T.; Miyashita, T.; Christensen, H.I. Navigation for human-robot interaction tasks. In Proceedings of the IEEE International Conference on Robotics and Automation ICRA '04, New Orleans, LA, USA, 26 April-1 May 2004; Volume 2, pp. 1894-1900. 
29. Gómez, J.V.; Mavridis, N.; Garrido, S. Social Path Planning: Generic Human-Robot Interaction Framework for Robotic Navigation Tasks. In Proceedings of the 2nd Intl. Workshop on Cognitive Robotics Systems: Replicating Human Actions and Activities, Tokyo, Japan, 3 November 2013.

30. Rios-Martinez, J. Socially-Aware Robot Navigation: Combining Risk Assessment and Social Conventions. Ph.D. Thesis, University of Grenoble, Saint-Martin-d'Heres, France, 2013.

31. Kosiński, T.; Obaid, M.; Woźniak, P.W.; Fjeld, M.; Kucharski, J. A fuzzy data-based model for Human-Robot Proxemics. In Proceedings of the 2016 25th IEEE International Symposium on Robot and Human Interactive Communication (RO-MAN), New York, NY, USA, 26-31 August 2016; pp. 335-340.

32. Sisbot, E.A.; Alami, R.; Simeon, T.; Dautenhahn, K.; Walters, M.; Woods, S. Navigation in the presence of humans. In Proceedings of the 5th IEEE-RAS International Conference on Humanoid Robots, Tsukuba, Japan, 5 December 2005; pp. 181-188. [CrossRef]

33. Sisbot, E.A.; Marin-Urias, L.F.; Alami, R.; Simeon, T. A Human Aware Mobile Robot Motion Planner. IEEE Trans. Robot. 2007, 23, 874-883. [CrossRef]

34. Satake, S.; Kanda, T.; Glas, D.F.; Imai, M.; Ishiguro, H.; Hagita, N. How to approach humans?-Strategies for social robots to initiate interaction. In Proceedings of the Conference on Human-Robot Interaction (HRI), 2009 4th ACM/IEEE International, La Jolla, CA, USA, 9-13 March 2009; pp. 109-116. [CrossRef]

35. Papadakis, P.; Spalanzani, A.; Laugier, C. Social mapping of human-populated environments by implicit function learning. In Proceedings of the 2013 IEEE/RSJ International Conference on Intelligent Robots and Systems, Tokyo, Japan, 3-7 November 2013; pp. 1701-1706.

36. Papadakis, P.; Rives, P.; Spalanzani, A. Adaptive spacing in human-robot interactions. In Proceedings of the 2014 IEEE/RSJ International Conference on Intelligent Robots and Systems, Chicago, IL, USA, 14-18 September 2014; pp. 2627-2632.

37. Charalampous, K.; Kostavelis, I.; Gasteratos, A. Robot navigation in large-scale social maps: An action recognition approach. Expert Syst. Appl. 2016, 66, 261-273. [CrossRef]

38. Walters, M.L.; Dautenhahn, K.; Te Boekhorst, R.; Koay, K.L.; Syrdal, D.S.; Nehaniv, C.L. An empirical framework for human-robot proxemics. In Procs of New Frontiers in Human-Robot Interaction; symposium at the AISB09 convention; 2009; pp. 144-149. Available online: https:/ / uhra.herts.ac.uk/handle/2299/9670 (accessed on 6 March 2020).

39. Mead, R.; Mataric, M.J. Probabilistic models of proxemics for spatially situated communication in hri. In Proceedings of the 2014 9th ACM/IEEE International Conference on Human-Robot Interaction (HRI), Bielefeld, Germany, 3-6 March 2014.

(c) 2020 by the authors. Licensee MDPI, Basel, Switzerland. This article is an open access article distributed under the terms and conditions of the Creative Commons Attribution (CC BY) license (http://creativecommons.org/licenses/by/4.0/). 